\title{
Riqueza de formigas (Hymenoptera: Formicidae) da Bacia da Sanga Caramuru, município de Chapecó-SC
}

\author{
Cristiano Ilha ${ }^{1 *}$ \\ Junir Antonio Lutinski ${ }^{1}$ \\ Dayana Von Muller Pereira \\ Flávio Roberto Mello Garcia ${ }^{2}$ \\ ${ }^{1}$ Universidade Comunitária Regional de Chapecó, UNOCHAPECÓ \\ Caixa Postal 747, CEP 89809-000, Chapecó - SC, Brasil \\ ${ }^{2}$ Universidade Federal de Pelotas, Departamento de Zoologia e Genética \\ Lab. de Ecologia de Insetos, Pelotas - RS, Brasil \\ *Autor para correspondência \\ cristiano_ilh@hotmail.com
}

Submetido em 05/02/2009

Aceito para publicação em 07/07/2009

\section{Resumo}

O presente estudo objetivou conhecer a riqueza da mirmecofauna associada à microbacia da Sanga Caramuru, no município de Chapecó, que representa um dos principais afluentes da barragem do Lajeado São José. As coletas ocorreram de junho de 2006 a maio de 2007. Realizaram-se coletas quinzenais, totalizando 21 coletas. Foram utilizadas armadilhas do tipo pit-fall. A análise dos dados foi realizada através do programa estatístico EstimateS (Colwell). Obtiveram-se 676 registros de espécies nas armadilhas. Foram identificadas 51 espécies distribuídas em 18 gêneros distribuídos em sete subfamílias. A riqueza estimada através do estimador Chao 2 foi de 61,6 espécies para a área de mata nativa, 42,3 espécies para a área de banhado e de 39,8 espécies para a área com eucalipto. O índice de diversidade de Shannon-Wiener obtido foi o mesmo $(3,2)$ para os ambientes amostrados. A maior riqueza e diversidade estimadas para a área de mata nativa coincidem com a maior riqueza também observada neste local. Embora os impactos da antropização sejam facilmente constatados na área estudada, os pequenos fragmentos de áreas com vegetação presentes neste local oferecem os recursos para a manutenção de uma significativa mirmecofauna.

Unitermos: Chao 2, Eucaliptus grandis, mirmecofauna, pit-fall, reflorestamento

\section{Abstract}

Richness of ants (Hymenoptera: Formicidae) in the Sanga Caramuru basin, Chapecó, SC. The present study aimed to elucidate the richness of ant fauna associated with the micro basin of Sanga Caramuru in the town of Chapecó that represents one of the principal tributaries of the São José dam. A total of 21 biweekly collections were made from June 2006 until May 2007, using Pit-fall traps. Analyzes of the data were carried out by the EstimateS statistics program (Colwell). A total of 676 registers of species were obtained through the traps. The richness obtained was 51 species distributed in 18 genera grouped in seven subfamilies. The richness estimated through the estimator Chao 2 was 61.6 species for the area of native forest, 42.3 species for the area of marsh, and 39.8 species for the area with eucalyptus. The Shannon and Wiener index obtained was 3.2 for the three sampled areas. The greater diversity estimated for the area with native forest coincided with the greater diversity also observed. Although the human impacts are easily evidenced in the studied area, the small fragments of areas with vegetation offer adequate resources for the maintenance of significant ant fauna.

Key words: ant fauna, Chao 2, Eucalytpus grandis, pit-fall, reforestation 


\section{Introdução}

Localizada na região oeste de Santa Catarina, município de Chapecó, a Sanga Caramuru é um importante afluente da barragem de captação de água que abastece toda a cidade. Esta é uma área que tem sofrido forte ação antrópica no decorrer dos anos, principalmente pelo desenvolvimento da agricultura, pastagens, silvicultura e, mais recentemente, pela urbanização. Essas ações tendem afetar a composição da fauna local, e um impacto deve ser percebido na estrutura de comunidades de comunidades de insetos que habitam esses ambientes.

As formigas formam um dos mais bem sucedidos grupos de insetos, sendo dominantes na maioria dos ecossistemas terrestres (Wilson, 1971). Muitas espécies de formigas são detritívoras e/ou predadoras de outros insetos, contribuindo para a reciclagem dos nutrientes e influenciando a dinâmica populacional de insetos herbívoros (Hölldobler e Wilson, 1990).

São conhecidas 342 espécies de formigas para o Estado de Santa Catarina, sendo que na região oeste são encontradas 179 espécies (Silva, 1999). A mirmecofauna desta região do Estado é relativamente bem conhecida, devido principalmente às coletas do naturalista Fritz Plaumann. Algumas pesquisas foram realizadas recentemente nesta região com formigas associadas a ambientes urbanos (Farneda et al., 2007; Iop et al., 2009) e na Floresta Nacional de Chapecó (Lutinski et al., 2008), que possui, além de mata nativa, áreas de reflorestamento.

Apesar de apresentar ecossistema predominante agrícola, a região da sanga Caramuru também apresenta confrontação geográfica com o ambiente urbano. Ambientes urbanos são geralmente reconhecidos como sendo áreas em profunda e constante atividade humana local, compostos de alta densidade de moradias humanas, estabelecimentos industriais e centros comerciais (McIntyre et al., 2001). O processo de urbanização pode afetar a distribuição de organismos de diversas formas, tornando-se uma ambiente onde muitas espécies alcançam altas densidades e assumem posições dominantes devido à existência de nichos desocupados, ausência de concorrentes e poucos inimigos naturais (Luck e Dahsten, 1974).
No Brasil, o processo de urbanização está ocorrendo em um ritmo acelerado, e muitas vezes avançando sobre áreas que são consideradas prioritárias para a conservação (Kamura et al., 2007). Estudos sobre a estrutura de comunidades de formigas vêm recebendo particular interesse, dada sua importância em estudos de impacto ambiental, servindo como bioindicadoras (Smith et al., 1992). Merecem este destaque por serem fáceis de coletar, relativamente fáceis de separar em morfoespécies e, serem sensíveis a mudanças no ambiente, apresentarem abundância local alta e também riqueza local alta, além de apresentarem muitos táxons especializados (Vasconcelos, 1998; Silva e Brandão, 1999).

O presente estudo objetivou conhecer a riqueza e a estrutura da comunidade de formigas associadas à microbacia da Sanga Caramuru no município de Chapecó. Inventários faunísticos são importantes para estabelecer uma base cientifica firme, para compreensão da biodiversidade e para implementação de planos de ocupação e manejo ambiental (Castri et al., 1992).

\section{Material e Métodos}

A Sanga Caramuru encontra-se no município de Chapecó, região oeste do Estado de Santa Catarina, cuja nascente e foz se localizam no território do próprio município $\left(27^{\circ} 05^{\prime} 02,58^{\prime}\right.$ 'S, 5240'00,05’O). Trata-se de uma área altamente antropizada através da agricultura, fruticultura, pastagem, silvicultura e área de camping. Apresenta alguns pequenos fragmentos florestais descontínuos (Figura 1).

Segundo a classificação de Köppen, o clima da região na qual a microbacia está inserida é do tipo mesotérmico, subtropical úmido, sem estação seca definida e com geadas severas frequentes (Cfa). As temperaturas médias mínimas e máximas variam entre $10,1^{\circ} \mathrm{C}$ e $19^{\circ} \mathrm{C}$ durante o ano. A umidade relativa do ar média é de $82,8 \%$ e a precipitação média anual é de aproximadamente $2.460 \mathrm{~mm}$.

As coletas foram realizadas em três regiões da microbacia:

Área A: Com extensão aproximada de 10ha, constituída por uma plantação de eucalipto (Eucalyptus 


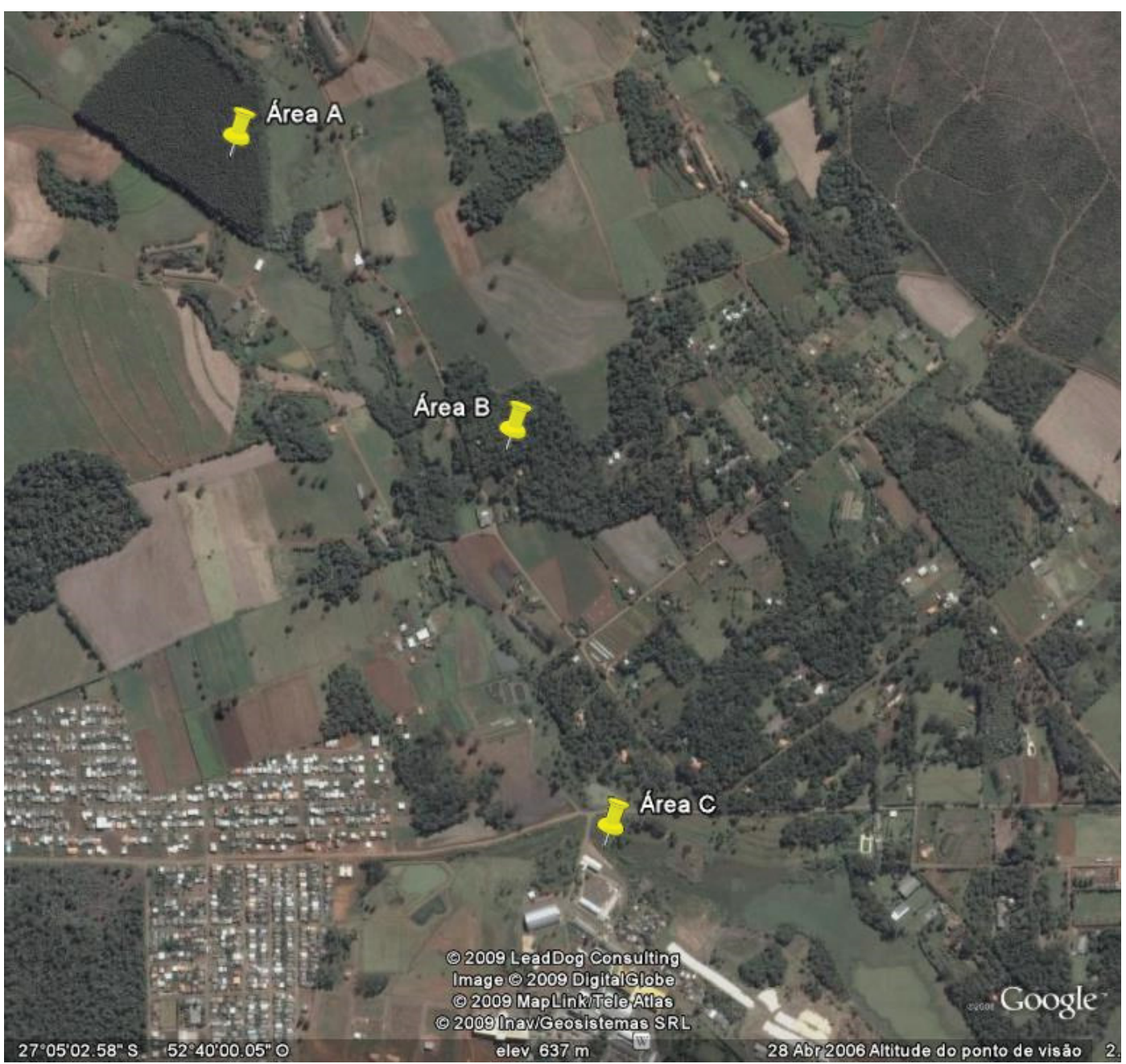

FIGURA 1: Distribuição das áreas amostrais na bacia da Sanga Caramuru selecionadas para inventário de formigas, no período de junho de 2006 a maio de 2007.

grandis Hill). Com idade de cinco anos, está localizada a uma distância de $1,5 \mathrm{~km}$ da área urbana. O sub-bosque verificado é composto essencialmente por gramíneas e arbustos esparsos, o que resulta em uma serapilheira muito pobre.

Área B: Com extensão de 8 ha, constitui um fragmento isolado de mata nativa, do tipo Floresta Ombrófila Mista, cercada por pastagens, com um dossel médio de $15 \mathrm{~m}$, sem histórico de desmatamento, a uma distância de $1 \mathrm{~km}$ da área urbana. Apresenta sub-bosque bem constituído e serapilheira rica, porém habitada por animais domésticos (galinhas e porcos).

Área C: Esta área constitui um banhado próximo à foz da Sanga na barragem do Lajeado São José. Apresenta vegetação arbustiva, com predominância de gramíneas. O solo caracteriza-se por ser úmido (encharcado), sem a presença de serapilheira e com alagamentos em períodos de chuvas prolongadas. Está inserido no perímetro urbano do município de Chapecó.

Coletas quinzenais foram realizadas durante o período de junho de 2006 a maio de 2007, totalizando 21 períodos de amostragem.

Foram utilizadas armadilhas do tipo pit-fall, com $10 \mathrm{~cm}$ de diâmetro e $20 \mathrm{~cm}$ de profundidade. Foram instaladas cinco armadilhas em cada área. Estas foram distribuídas em transectos lineares perpendiculares à borda ficando a primeira armadilha a cinco metros da borda e seguindo a intervalos de $10 \mathrm{~m}$ uma da outra. As 
armadilhas continham uma solução de $200 \mathrm{ml}$ de água com uma gota de detergente, para quebrar a tensão superficial. As armadilhas permaneciam nas áreas, sendo revisadas, a cada 15 dias entre as coletas.

Portanto, cada coleta representou o esforço amostral de 15 armadilhas por dia, sendo cinco em cada área avaliada. $\mathrm{O}$ esforço amostral total gerado no estudo foi de 315 inspeções em armadilhas durante o período de estudo, ou seja, 15 armadilhas instaladas e inspecionadas 21 vezes (coletas).

Os espécimes coletados foram armazenados em frascos contendo álcool 70\%, devidamente etiquetados com os dados da coleta. Posteriormente, foram transportados para o Laboratório de Entomologia da Universidade Comunitária Regional de Chapecó UNOCHAPECÓ para triagem e identificação. A triagem do material se realizou pela separação em morfoespécies, que posteriormente foram identificadas até gênero segundo chaves propostas por Palacio e Fernández (2003) e até espécie através de consulta aos espécimes depositados no Museu Zoobotânico da Universidade Comunitária Regional de Chapecó - Unochapecó, e classificadas conforme Bolton (2003).

Todos os parâmetros das análises estatísticas foram obtidos a partir de equações aplicadas sobre o número de registros feitos para cada espécie durante as coletas. A frequência relativa foi calculada pela equação $\mathrm{F}(\%)=$ $\mathrm{F}_{\mathrm{i}} / \mathrm{F}_{\mathrm{t}} \mathrm{X} 100$, onde $\mathrm{F}_{\mathrm{i}}$ é o número de registros da espécie em uma determinada área e $\mathrm{F}_{\mathrm{t}}$ é o número de registros totais para aquela área. Para evitar a superestimação de espécies com colônias localizadas nas proximidades dos pit-falls, evitou-se análises estatísticas sobre o número de espécimes coletados. O índice de diversidade de Shannon e Wiener (H') foi obtido seguindo a proposta de Magurran (1988).

Foram construídas curvas de riqueza através do estimador Chao2. Este indicador foi obtido através do programa EstimateS 6.0.b1 (Colwell, 2000). A similaridade entre as três áreas foi avaliada a partir de uma análise de agrupamento com associação completa dos dados obtida através do método Bray-Curtis com análise de Cluster (Krebs, 1989), utilizando o programa
BioDiversity Pro Versão 2. Através deste mesmo programa foram calculadas as correlações entre a riqueza mensal obtida e os fatores climáticos da temperatura mensal média e da pluviosidade mensal média tendo como parâmetro a correlação de Pearson e a significância de $\mathrm{p}<0,05$. Os dados meteorológicos foram obtidos através da EPAGRI cuja estação de coleta dos dados encontra-se localizada há aproximadamente $4 \mathrm{~km}$ da área amostrada.

\section{Resultados}

Foram capturadas 5.836 formigas com 676 registros de espécies obtidos nas amostras. A riqueza observada foi de 51 espécies, distribuídas em 18 gêneros e oito subfamílias: Dolichoderinae, Ectatomminae, Ecitoninae, Formicinae, Myrmicinae, Heteroponerinae, Ponerinae e Pseudomyrmecinae (Tabela 1).

Foram registradas na Área A, 34 espécies, sendo as mais frequentes: Camponotus rufipes (Fabricius 1775) (25,3\%), Atta sexdens Linnaeus, 1758 (11,5\%) e Labidus coecus (Latreille,1802) (10\%) Sendo que as espécies que ocorreram somente nesta área foram: Solenopsis sp. 1 (1,1\%), Pseudomyrmex gracilis (Fabricius, 1804) (2\%), Tapinoma melanocephalum Fabricius, 1793 (0,4\%), Camponotus sp. $2(0,4 \%)$ e Camponotus sp. $7(0,4 \%)$. (Tabela 1)

$\mathrm{Na}$ Área B foram registradas 37 espécies, sendo a espécie de maior frequência, L. coecus $(22,7 \%)$. As espécies encontradas exclusivamente neste ponto amostral foram: Crematogaster nigropilosa Mayr, 1870 (0,5\%), Pachycondyla sp. 1 (0,5\%), Acanthoponera mucronata (Roger, 1860) (0,5\%), Hypoponera foeda (Forel, 1912) (0,5\%), Gnamptogenys sp. (0,5\%) Camponotus sericeiventris Guérin-Méneville, 1838 (0,5\%) Camponotus sp. $3(0,5 \%)$ e Camponotus sp. 5 (1\%). (Tabela 1).

$\mathrm{Na}$ Área $\mathrm{C}$ foram registradas 36 espécies com maior numero de registros para L. coecus (15\%), C. rufipes (17,4\%). Exclusivamente neste local foram encontradas: Hypoponera opacior (Forel, 1893) (0,5\%), Camponotus sp. $6(1,4 \%)$ e Camponotus sp. $8(0,5 \%)$. 
TABELA 1: Número de registros e frequências relativas das formigas coletadas, na área de influências da Sanga Caramuru, município de Chapecó, Santa Catarina, no período de junho de 2006 a maio de 2007.

\begin{tabular}{|c|c|c|c|c|c|c|}
\hline \multirow[t]{2}{*}{ Táxon } & \multicolumn{6}{|c|}{ Registros e frequências } \\
\hline & Área A & $(\%)$ & Área B & $(\%)$ & Área C & $(\%)$ \\
\hline \multicolumn{7}{|l|}{ Subfamília Dolichoderinae } \\
\hline \multicolumn{7}{|l|}{ Tribo Dolichoderini } \\
\hline Dorymyrmex sp. & - & - & 5 & 2,6 & 3 & 1,4 \\
\hline Linepithema humile Mayr, 1868 & - & - & 5 & 2,6 & 2 & 0,9 \\
\hline Tapinoma melanocephalus Fabricius, 1793 & 1 & 0,4 & - & - & - & - \\
\hline \multicolumn{7}{|l|}{ Subfamília Ecitoninae } \\
\hline \multicolumn{7}{|l|}{ Tribo Ecitonini } \\
\hline Labidus coecus (Latreille, 1802) & 27 & 10 & 44 & 22,7 & 32 & 15 \\
\hline Labidus praedator (Fr. Smith, 1858) & 14 & 5,2 & 4 & 2,1 & 1 & 0,5 \\
\hline \multicolumn{7}{|l|}{ Subfamília Ectatomminae } \\
\hline \multicolumn{7}{|l|}{ Tribo Ectatommini } \\
\hline Gnamptogenys striatula Mayr, 1884 & 2 & 0,7 & 8 & 4,1 & 2 & 0,9 \\
\hline Gnamptogenys sp. & - & - & 1 & 0,5 & - & - \\
\hline \multicolumn{7}{|l|}{ Subfamília Formicine } \\
\hline \multicolumn{7}{|l|}{ Tribo Camponotini } \\
\hline Camponotus crassus Mayr, 1862 & 14 & 5,2 & - & - & 17 & 8 \\
\hline Camponotus diversipalpus Santschi, 1922 & 7 & 2,6 & 6 & 3,1 & 6 & 2,8 \\
\hline Camponotus rufipes (Fabricius, 1775) & 68 & 25,3 & 6 & 3,1 & 37 & 17,4 \\
\hline Camponotus sericeiventris G-Mén., 1838 & - & - & 1 & 0,5 & - & - \\
\hline Camponotus sp.1 & 4 & 1,5 & 1 & 0,5 & 1 & 0,5 \\
\hline Camponotus sp.2 & 1 & 0,4 & - & - & - & - \\
\hline Camponotus sp.3 & & & 1 & 0,5 & - & - \\
\hline Camponotus sp.4 & 2 & 0,7 & - & - & 2 & 0,9 \\
\hline Camponotus sp.5 & - & - & 2 & 1 & - & - \\
\hline Camponotus sp.6 & - & - & - & - & 3 & 1,4 \\
\hline Camponotus sp.7 & 1 & 0,4 & - & - & - & - \\
\hline Camponotus sp. 8 & - & - & - & - & 1 & 0,5 \\
\hline \multicolumn{7}{|l|}{ Tribo Plagiolepidini } \\
\hline Paratrechina fulva (Mayr, 1862) & - & - & 3 & 1,7 & 1 & 0,5 \\
\hline Paratrechina longicornis Latreille, 1802 & 7 & 2,6 & 9 & 4,6 & 14 & 6,6 \\
\hline Paratrechina sp. & 6 & 2,2 & 1 & 0,5 & 3 & 1,4 \\
\hline \multicolumn{7}{|l|}{ Subfamília Heteroponerinae } \\
\hline \multicolumn{7}{|l|}{ TriboHeteroponerini } \\
\hline Acanthoponera mucronata (Roger, 1860) & - & - & 1 & 0,5 & - & - \\
\hline Heteroponera microps Borgmeier, 1957 & - & - & - & - & 1 & 0,5 \\
\hline \multicolumn{7}{|l|}{ Subfamília Myrmicinae } \\
\hline \multicolumn{7}{|l|}{ Tribo Crematogastrini } \\
\hline Crematogaster corticicola Mayr, 1887 & 2 & 0,7 & 12 & 6,2 & 2 & 0,9 \\
\hline
\end{tabular}


Crematogaster nigropilosa Mayr, 1870

$\begin{array}{llll}- & - & 1 & 0,5\end{array}$

\begin{tabular}{|c|c|c|c|c|c|c|}
\hline \multicolumn{7}{|l|}{ Tribo Pheidolini } \\
\hline Pheidole sp.1 & 11 & 4,1 & 11 & 5,7 & 13 & 6,1 \\
\hline Pheidole sp. 2 & 10 & 3,7 & 2 & 1 & 4 & 1,8 \\
\hline Pheidole sp.3 & 3 & 1,1 & - & - & 1 & 0,5 \\
\hline Pheidole sp.4 & 8 & 3 & 6 & 3,1 & 4 & 1,88 \\
\hline Pheidole sp.5 & - & - & 1 & 0,5 & - & - \\
\hline \multicolumn{7}{|l|}{ Tribo Solenopsidini } \\
\hline Solenopsis saevissima (Fr.Smith, 1855) & 2 & 0,7 & - & - & 3 & 1,4 \\
\hline Solenopsis sp.1 & 3 & 1,1 & - & - & - & - \\
\hline Solenopsis sp.3 & 3 & 1,1 & 1 & 0,5 & 1 & 0,5 \\
\hline \multicolumn{7}{|l|}{ Tribo Attini } \\
\hline Acromyrmex disciger (Mayr, 1887) & 6 & 2,2 & 8 & 4,1 & 7 & 3,3 \\
\hline Acromyrmex niger (Fr. Smith, 1858) & 3 & 1,1 & 5 & 2,6 & 9 & 4,2 \\
\hline Acromyrmex subterraneus Forel, 1893 & 7 & 2,6 & 16 & 8,2 & 3 & 1,4 \\
\hline Acromyrmex sp. & 6 & 2,2 & 1 & 0,5 & 3 & 1,4 \\
\hline Atta sexdens Linnaeus, 1758 & 31 & 11,5 & 2 & 1 & 17 & 8 \\
\hline Mycocepurus goeldii Forel, 1893 & 1 & 0,4 & 1 & 0,5 & 1 & 0,5 \\
\hline \multicolumn{7}{|l|}{ Subfamília Ponerinae } \\
\hline \multicolumn{7}{|l|}{ Tribo Ponerini } \\
\hline Dinoponera australis Emery, 1910 & - & - & 1 & 0,5 & 7 & 3,3 \\
\hline Hypoponera foeda (Forel, 1912) & - & - & 1 & 0,5 & - & - \\
\hline Hypoponera opacior (Forel, 1893) & - & - & - & - & 1 & 0,5 \\
\hline Pachycondyla crenata (Roger, 1861) & 1 & 0,4 & 1 & 0,5 & - & - \\
\hline Pachycondyla harpax (Fabricius, 1804) & 2 & 0,7 & 13 & 6,7 & 1 & 0,5 \\
\hline Pachycondyla striata Fr. Smith, 1858 & 3 & 1,1 & 8 & 4,1 & 3 & 1,4 \\
\hline Pachycondyla villosa (Fabricius, 1804) & 5 & 2 & 3 & 1,7 & 3 & 1,4 \\
\hline Pachycondyla sp. 1 & - & - & 1 & 0,5 & - & - \\
\hline \multicolumn{7}{|l|}{ Subfamília Pseudomyrmecinae } \\
\hline \multicolumn{7}{|l|}{ Tribo Pseudomyrmecini } \\
\hline Pseudomyrmex gracilis (Fabricius, 1804) & 5 & 2 & 0 & 0 & 1 & 0,5 \\
\hline Pseudomyrmex pallidus (Fr. Smith, 1855) & 1 & 0,4 & 1 & 0,5 & 0 & 0 \\
\hline Pseudomyrmex sp. & 2 & 0,7 & 0 & 0 & 3 & 1,4 \\
\hline Subtotais & 269 & 100 & 194 & 100 & 213 & 100 \\
\hline Total de registros & \multicolumn{6}{|c|}{676} \\
\hline
\end{tabular}

O número de espécies de formigas não estabilizou completamente em nenhuma das áreas ao final das coletas embora a área que tenha demonstrado maior estabilização da riqueza encontrada foi a área $\mathrm{A}$ (Figura 2).

Os índices de diversidade de Shannon e Wiener encontrados para as três áreas apresentam o mesmo valor
$(3,2)$, entretanto o estimador de riqueza Chao2 estimou maior riqueza para a área $\mathrm{B}$. A área $\mathrm{A}$ foi a que apresentou a riqueza observada mais próxima da estimada (Figura 3). As áreas $\mathrm{A} \mathrm{e} \mathrm{C}$ apresentaram uma maior similaridade em relação a área B (Figura 4). 


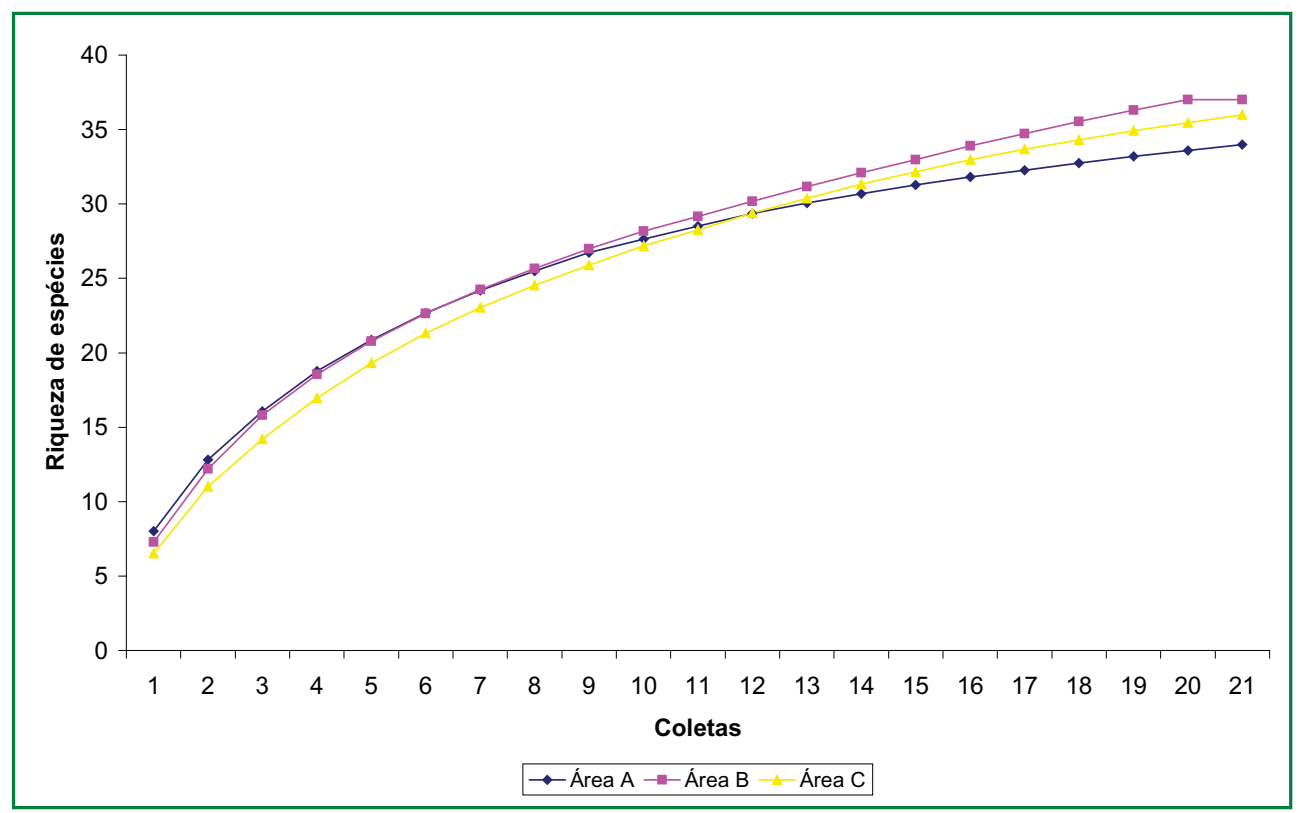

FIGURA 2: Riqueza de formigas observada em três áreas de influência da Sanga Caramuru, município de Chapecó, Santa Catarina, no período de junho de 2006 a maio de 2007.

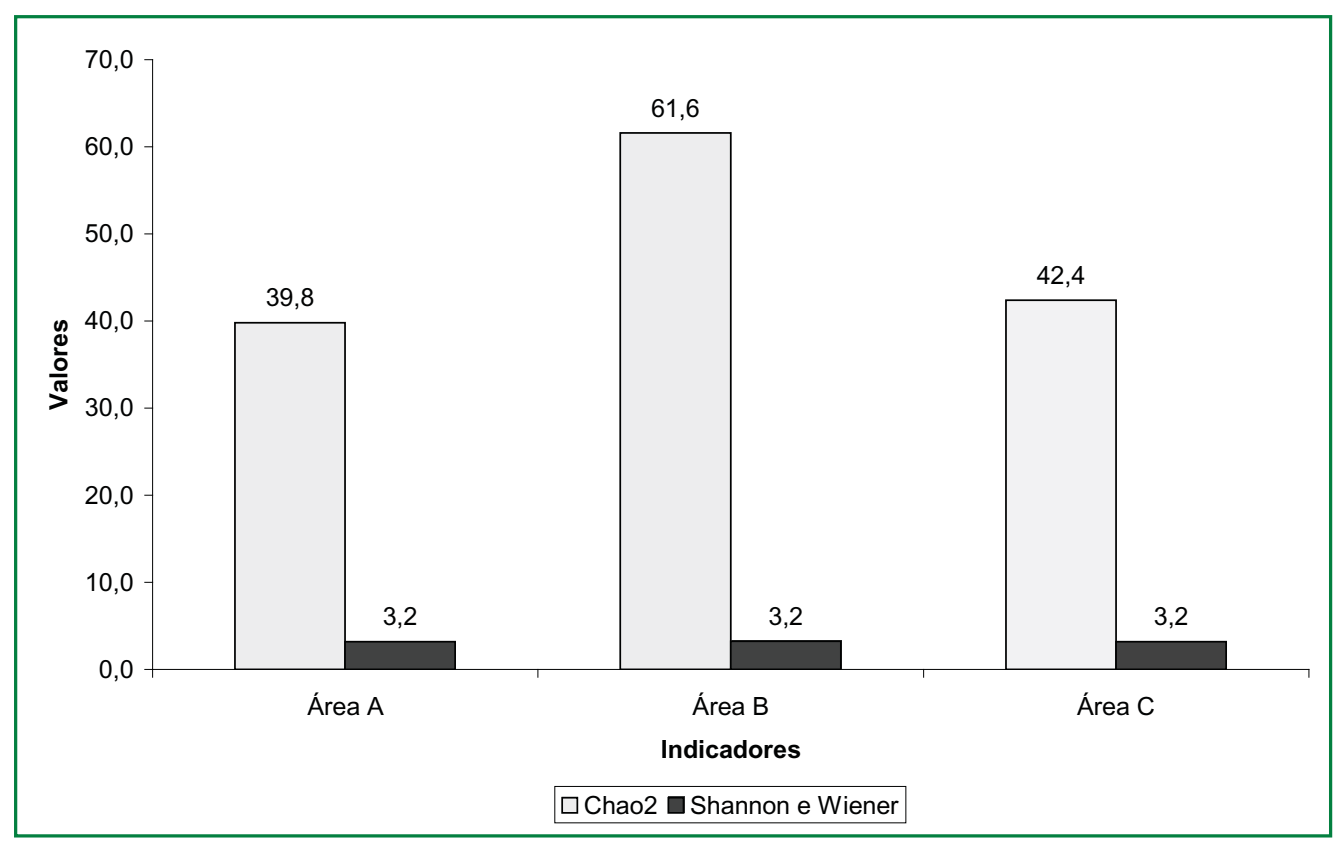

FIGURA 3: Riqueza de formigas estimada (Chao 2) e índice de diversidade (Shannon e Wiener) em três áreas de influência da Sanga Caramuru, município de Chapecó, Santa Catarina, no período de junho de 2006 a maio de 2007. 


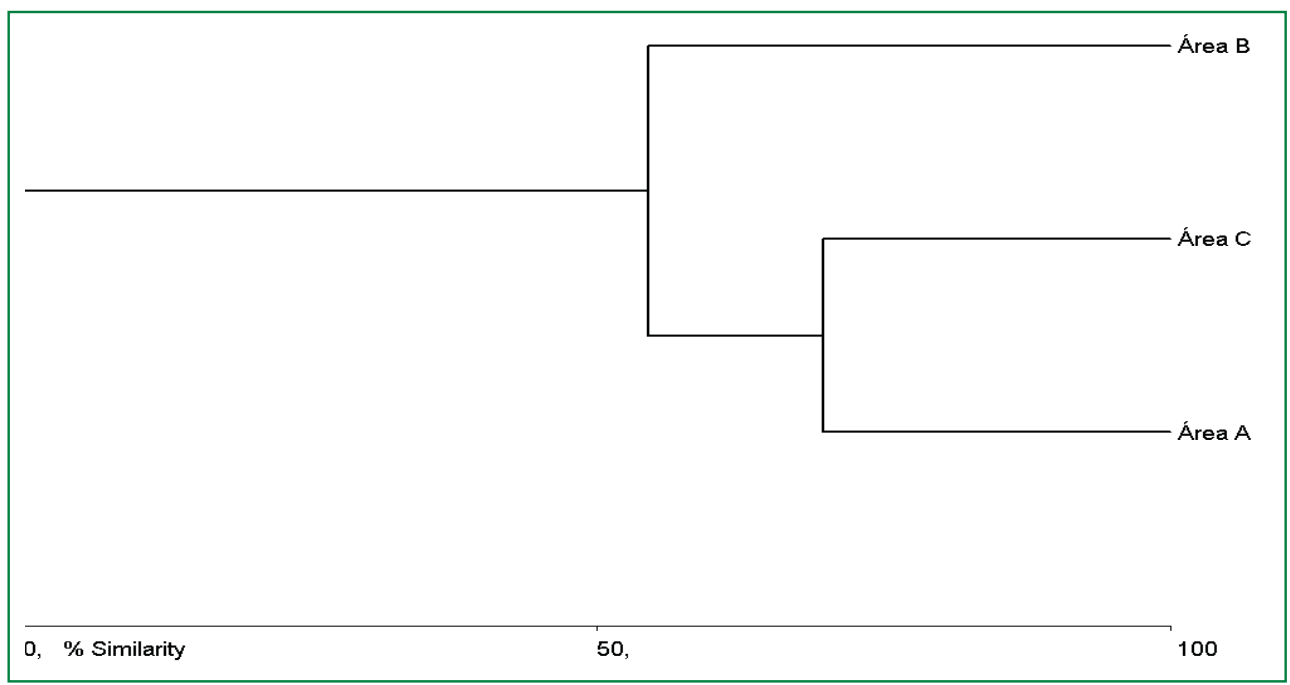

FIGURA 4: Similaridade entre a fauna de formigas de três áreas da bacia da Sanga Caramuru, município de Chapecó, no período de junho de 2006 a maio de 2007 (Bray-Curtis Cluster Analysis).

Quanto á influência dos fatores climáticos sobre as coletas, observou-se uma forte correlação com as temperaturas médias mensais, enquanto que para os índices de pluviosidade mensais a correlação foi menor (Tabela 2).

TABELA 2: Correlação entre a riqueza de formigas observadas mensalmente na bacia da Sanga Caramuru (S(obs)) e os fatores climáticos de pluviosidade e temperaturas médias mensais, no município de Chapecó, no período de junho de 2006 a maio de 2007.

\begin{tabular}{cccc}
\hline Mês & S(obs) & $\begin{array}{c}\text { Pluviosidade } \\
(\mathbf{m m}) *\end{array}$ & $\begin{array}{c}\text { Temperatura média } \\
\text { mensal }\left({ }^{\circ} \mathbf{C}\right) *\end{array}$ \\
\hline jun/06 & 17 & 114,3 & 16,7 \\
jul/06 & 13 & 62,8 & 17,0 \\
ago/06 & 18 & 125,2 & 16,6 \\
set/06 & 13 & 156,5 & 17,0 \\
out/06 & 25 & 96,2 & 21,7 \\
nov/06 & 23 & 223,3 & 21,4 \\
dez/06 & 28 & 236,3 & 24,4 \\
jan/07 & 23 & 115,9 & 24,1 \\
fev/07 & 34 & 231,7 & 23,4 \\
mar/07 & 30 & 152,6 & 23,4 \\
abr/07 & 26 & 296,9 & 20,3 \\
mai/07 & 20 & 327,8 & 14,0 \\
\hline \multicolumn{7}{c}{ Coeficiente de } \\
correlação ( r ) & $\mathbf{0 , 4 1 * *}$ & $\mathbf{0 , 7 8 * *}$ \\
\hline
\end{tabular}

* Fonte: EPAGRI; ** Correlação significante para $p<0,05(\mathrm{Pe}-$ arson)

\section{Discussão}

O fragmento de mata nativa (área B) foi o que apresentou um maior número de espécies exclusivas. Pode-se inferir que, em um ambiente com forte ação antrópica, essa área representa um importante remanescente para muitas espécies que são sensíveis a perturbações ambientais. Ambientes mais complexos estruturalmente como a área de mata nativa (área B) podem manter maior diversidade e riqueza por apresentarem maiores possibilidades de abrigo, reprodução, nidificação e alimentação, além de maior estabilidade (Oliveira et al., 1995).

A subfamília Dolichoderinae foi representada por três espécies: Dorymyrmex sp., Linepithema humile Mayr, 1868 e T. melanocephalum. Uma espécie foi registrada apenas na plantação de eucalipto (Área A) (Tapinoma melanocephalum) enquanto que as demais obtiveram registros nas Áreas de mata nativa (Área B) e banhado (Área C). Tapinoma melanocephalum é popularmente conhecida como formiga fantasma e geralmente encontrada em ambientes urbanos, associadas às zonas de comércio (Brooks et al., 2008).

A espécie com maior número de registros da subfamília Ecitoninae foi $L$. coecus, sendo encontrada em todas as áreas. Espécies pertencentes a esta subfamília, caracterizam-se por possuírem hábitos predatórios, 
nômades, com fases estacionárias e migratórias, (Palacio, 2003). Lutinski et al. (2008), em seu trabalho na floresta Nacional de Chapecó, constataram que essa espécie demonstra tolerância a perturbações ambientais como as observadas nas áreas A e B.

A subfamília Formicinae está representada por duas tribos, nesse trabalho: Camponotini e Plagiolepidini.

A tribo Camponotini foi a que apresentou o maior número de espécies, totalizando 12. Pode-se destacar $C$. rufipes como a espécie com maior número de registros e Camponotus crassus Mayr, 1862, que também foi bem representada. Estas duas espécies foram pouco frequentes na mata nativa (Área B). Resultado semelhante a esse foi encontrado por Lutinski et al. (2008), onde essas espécies apresentaram maior frequência nas monoculturas de eucalipto e Pinus sp. em relação a mata nativa. Com esses dados é possível constatar que se trata de espécies que possuem preferência por habitat de áreas abertas.

O gênero Paratrechina foi o único observado para a tribo Plagiolepidini e obteve registros em todas as áreas. Segundo Fernández (2003), essas espécies são comuns tanto em ambientes naturais quanto em ambientes que sofreram algum tipo de distúrbio, justificando o motivo pelo qual essa espécie obteve registros em todas as áreas.

A subfamília Myrmicinae foi a que apresentou o maior número de espécies nos três pontos amostrais, com 16 espécies distribuídas em quatro tribos.

Destacaram-se os registros de Atta sexdens (Tribo Attini), sendo encontrada nas três áreas, mas de forma pouco frequente na área de mata nativa. Essa espécie é considerada uma praga, para a silvicultura. (Oliveira et al., 2004). Também da Tribo Attini destacou-se $A$. subterraneus, sendo registrada nas três áreas, mas com maior número de registros na área B. Essa espécie está geralmente associada a áreas florestadas (Delabie e Fowler, 1995).

As formigas do gênero Atta e Acromyrmex são conhecidas como "formigas cortadeiras", sendo consideradas como importantes pragas de áreas de reflorestamento, pois atacam intensamente as plantas em qualquer fase de seu desenvolvimento, cortando suas folhas, flores, brotos. Por essas razões, essas formigas vêm sendo alvo de diversas pesquisas para o desenvolvimento de métodos para controle (Marinho et al., 2006).

A subfamilia Ponerinae foi representada por oito espécies. O gênero Pachycondyla obteve registros em todas as áreas, sendo $P$. harpax mais frequente na área de mata nativa. As espécies pertencentes a essa subfamília são comuns em ambientes úmidos e áreas que apresentam bosques (Lattke, 2003). Essa característica explica porque elas foram mais frequentes nas áreas de mata nativa e banhado.

Foram registradas três espécies de Pseudomyrmecinae pertencentes ao gênero Pseudomyrmex. Este apresentou poucos registros e isso pode ser devido ao hábito arborícola destas espécies que, consequentemente, estão menos propensas a caírem em pit-falls. Algumas espécies associam-se a cochonilhas, podendo causar danos a frutíferas (Ward, 2003).

A maior similaridade entre as áreas A e C evidenciada na Figura 4 pode ser atribuída a características ecológicas das espécies de formigas compartilhadas por estas duas áreas. Observa-se na Tabela 1 que C. crassus, Camponotus sp. 4, Camponotus sp. 6, Camponotus sp. 7, Camponotus sp. 8, Pheidole sp. 3 e S. saevissima foram encontradas somente nestas duas áreas. Segundo Silvestre et al. (2003) estas formigas possuem comportamento generalista e agressivo e encontram-se geralmente associadas a ambientes perturbados.

A maior frequência de espécies como C. rufipes e $L$. coecus na área A também corroboram a ideia de que esta área encontra-se em condições de perturbação ecológica. Ambas as espécies são frequentemente encontradas em ambientes abertos e pobres em vegetação, caracterizando-se assim como espécies generalistas. Resultados semelhantes foram obtidos para área de reflorestamento de eucalipto por Lutinski et al., 2008. A destacada frequência $A$. sexdens nesta mesma área pode ser explicada pela abundante oferta de alimento.

Com relação às estimativas de riqueza de espécies obtidas, as áreas A e C não apresentam diferença significativa entre si e tampouco em relação ás espécies encontradas. No entanto, pode-se notar que, na área B, 
a estimativa da diversidade de espécies de formigas foi $60 \%$ maior que a encontrada (Figura 3). Nesta observase um sub-bosque de idade avançada e, por isso, pode haver esta correlação. Segundo Marinho et al. (2002), existe uma correlação entre a riqueza de espécies de formigas e a de plantas, ou seja, a colonização de áreas por certas plantas é seguida pela colonização de espécies de formigas. Portanto, nesta área, o número de espécies tenderá a crescer com um incremento no esforço amostral (Figura 2).

O estimador Chao 2 é baseado na incidência, sendo influenciado pelas espécies que ocorrem em apenas uma amostra e por aquelas que ocorrem em apenas duas amostras (Chao, 1987). Este padrão foi observado na comunidade de formigas presente na área de mata nativa, onde 16 espécies obtiveram apenas um ou dois registros, demonstrando que esta área é composta por um número menor de espécies dominantes em relação ao que se observa nas demais áreas. Espécies como $A$. mucronata, H. foeda, Gnamptogenys sp. e Pachycondyla sp. 1 são predadoras (Silvestre et al., 2003) de outros insetos e, por isso, sua presença exclusivamente nesta área é um indicativo de maior complexidade estrutural e de interações desta comunidade.

Embora que Chao 2 tenha estimado um maior número de espécies para a área $\mathrm{B}, \mathrm{o}$ índice de diversidade de Shannon e Wiener apresentou os mesmos valores para as três áreas. Este índice leva em consideração o número de espécies (Schmidt et al., 2005) e considera igual peso às espécies raras e abundantes (Magurran, 1988). Considerando o valor de Shannon e Wiener obtido igualmente para as três áreas $(3,2)$ e comparado-o com índice encontrado para remanescente preservado da Floresta Nacional de Chapecó (FLONA) $(3,4)$ por Lutinski et al. (2008), pode-se inferir que a diversidade em cada área é significativa.

A correlação positiva da riqueza de espécies com a variável climática temperatura média pode ter ligação com uma maior atividade de forrageio das formigas em meses mais quentes. Chagas e Vasconcelos (2002) observaram que algumas espécies têm preferência por temperaturas entre $20^{\circ} \mathrm{C}$ e $29^{\circ} \mathrm{C}$, faixa de temperaturas médias observadas durante o período de outubro de 2006 a abril de 2007 quando observou-se uma maior riqueza mensal. Entretanto, com relação à pluviosidade, a correlação foi menor em relação a correlação com a temperatura média.

Embora Chagas e Vasconcelos (2002) tenham observado que algumas espécies continuam a forragear mesmo em dias de verão com pancadas de chuva, esta correlação no presente trabalho pode ter sido influenciada principalmente pelo período seco observado no mês de outubro de 2006, quando foi observada uma riqueza de 25 espécies, e pelo período chuvoso observado em maio de 2007, quando foram registradas apenas 20 espécies.

A partir destes dados, pode-se inferir que a comunidade de formigas da bacia da Sanga Caramuru está fortemente associada aos fragmentos remanescentes de vegetação. A remoção dos mesmos poderá fazer com que algumas espécies desapareçam localmente, principalmente nos fragmentos de mata nativa.

\section{Referências}

Bolton, B. 2003. Synopsis and classification of Formicidae. Memoirs of the American Entomological Institute, Gainesville, USA, 370pp.

Brooks, M. D.; Nentwig, G.; Gutsmann, V. 2008. Elimination of a Tapinoma Melanocephalum (Hymenoptera: Formicidae) infestation using imidadcloprid bait. Proceedings of the Sixth International Conference on Urban Pests, Veszprém, Hungria, p.219-223.

Castri, F.; Vernhes, J. R.; Younès, T. 1992. Inventorying and monitoring biodiversity: A proposal for an international network. Biology International, Special Issue, 27: 1-29.

Chagas, A. C. S.; Vasconcelo, V. O. 2002. Comparação da frequência da atividade forrageira da formiga Pachycondyla obscuricornis (Emery, 1890) (Hymenoptera, Formicidae) no verão e no inverno, em condições de campo. Revista Brasileira de Zoociências, 4 (1): 97-109.

Chao, A. 1987. Estimating the population size for capture-recapture data with unequal catchability. Biometrics, 43: 783-791.

Colwell, R. K. 2000. Estimates: Statistical estimations of species richness and shared species from samples. Version 6.0b1. User's guide and application published at http://viceroy.eeb.uconn.edu/ estimates.

Delabie, J. H. C.; Fowler, H. G. 1995. Soil and litter cryptic ant assemblages of Bahian cocoa plantations. Pedobiologia, 39: 423433

Farneda. F. Z.; Lutinski, J. A.; Garcia, F. R. M. 2007. Comunidade de formigas (Hymnoptera: Formicidae) na área urbana do município de Pinhalzinho, Santa Catarina, Brasil. Revista de Ciências Ambientais, 1: 53-56.

Fernández, F. 2003. Subfamilia Formicinae. In: Fernández, F. (Ed.). Introducción a las hormigas de la región Neotropical. Instituto 
de Investigación de Recursos Biológicos Alexander von Humboldt, Bogotá, Colombia, p.299-306.

Hölldobler, B.; Wilson, E. O. 1990. The ants. Belknap, Havard University Press, Cambridge, UK, 732pp.

Iop, S.; Caldart, V. M.; Lutinski, J. A.; Garcia, F. R. M. 2009. Formigas urbanas da cidade de Xanxerê, Santa Catarina, Brasil. Biotemas, 22 (2): 49-58.

Karamura, C. M.; Morini, M. S. C.; Figueiredo, C. J.; Bueno, O. C. B.; Campos-Farinha, A. E. C. 2007. Ant communities (Hymenoptera: Formicidae) in an urban ecosystem near the Atlantic Rainforest. Brazilian Journal of Biology, 67 (4): 635-641.

Krebs, C. J. 1989. Bray-Curtis Cluster Analysis. 1. Biodiversity Pro Versão 2. Disponível em $<$ http://biodiversity-pro.software. informer.com/>.

Lattke, J. E. 2003. Subfamilia Ponerinae. In: Fernández, F. (Ed.). Introducción a las hormigas de la región Neotropical. Instituto de Investigación de Recursos Biológicos Alexander von Humboldt, Bogotá, Colombia, p.261-276.

Luck, R. F.; Dahsten, D. L. 1974. Natural decline of a pine needle scale (Chionaspis pinifoliae (Fitch)) outbreak at South Lake Tahoe, California, following cessation of adult mosquito control with malathion. Ecology, 54 (4): 893-904.

Lutinski, J. A.; Garcia, F. R. M.; Lutinski, C. J.; Iop, S. 2008. Diversidade de formigas na Floresta Nacional de Chapecó, Santa Catarina, Brasil. Ciência Rural, 38 (7): 1810-1816.

Magurran, A. 1988. Ecological diversity and its measurement. Princeton University Press, New Jersey, USA, 75pp.

Marinho, C. G. S.; Della Lucia, T. M. C.; Picanço, M. C. 2006 Fatores que dificultam o controle das formigas cortadeiras. Bahia Agrí́cola, 7 (2): 18-21.

McIntyre, N. E.; Rangs, J.; Fagan, W. F.; Faeth, S. H. 2001. Ground arthropod community struture in a heterogeneous urban environment. Landscape and Urban Planning, 52 (4): 257-274.

Oliveira. H. G.; Lacerda, F. G.; Marinho, C. G. S.; Della Lucia, T. M. C. 2004. Atratividade de Atta sexdens rubropilosa por plantas de eucalipto atacadas previamente ou não por Thyrinteina arnobia. Pesquisa Agropecuária Brasileira, 39 (3): 285-287.

Oliveira. M. A.; Della Lucia, T. M. C; Araujo, M. S.; Cruz, A. D. A. 1995. A fauna de formigas em povoamentos de eucalipto e mata nativa no estado do Amapá. Acta Amazônica, 25 (1/2): 117-126.
Palacio, E. E. 2003. Subfamilia Ecitoninae. In Fernández, F. (Ed.). Introducción a las hormigas de la región Neotropical. Instituto de Investigación de Recursos Biológicos Alexander von Humboldt, Bogotá, Colombia, p.281-285.

Palacio, E. E.; Fernández, F. 2003. Clave para las subfamilias y gêneros. In: Fernández, F. (Ed.). Introducción a las hormigas de la región Neotropical. Instituto de Investigación de Recursos Biológicos Alexander von Humboldt, Bogotá, Colombia, p.233260.

Silva, R. R. 1999. Formigas (Hymenoptera: Formicidae) do oeste de Santa Catarina: Histórico das coletas e lista atualizada das espécies do Estado de Santa Catarina. Biotemas, 12 (2): 75-100.

Silva, R. R.; Brandão, C. R. F. 1999. Formigas (Hymnoptera: Formicidae) como indicadores da qualidade ambiental e da biodiversidade de outros invertebrados terrestres. Biotemas, 12 (2): 55-73.

Silvestre, R.; Brandão, C. R. F.; Silva, R. R. 2003. Grupos funcionales de hormigas: El caso de los gremios del cerrado. In: Fernández, F. (Ed.). Introducción a las hormigas de la región Neotropical. Instituto de Investigación de Recursos Biológicos Alexander von Humboldt, Bogotá, Colombia, p.113-148

Schmidt, K.; Corbetta, R.; Camargo, A. J. A. 2005. Formigas (Hymenoptera: Formicidae) da Ilha João da Cunha, SC: Composição e diversidade. Biotemas, 18 (1): 57-71.

Smith, M. R. B.; Delabie, J. H. C.; Carzola, I. M.; da Encarnação, A. M. Z.; Casimiro, A. B.; Nascimento, I. C.; Souza, M.; Furst A. L. B. 1992. Uso de formigas como bioindicadores: Primeiras indicações de padrões de interação entre vegetação, atividades agrícolas e comunidades de Formicidade. Resumos do XVI Congresso Latino-Americano e Brasileiro de Zoologia, Belém, Brasil, p.146.

Vasconcelos, H. C. 1998. Respostas das formigas a fragmentação florestal. Série Técnica Ipef, 12 (32): 95-98.

Ward, P. S. 2003. Subfamília Pseudomyrmecinae. In: Fernández, F. (Ed.). Introducción a las hormigas de la región Neotropical. Instituto de Investigación de Recursos Biológicos Alexander von Humboldt, Bogotá, Colombia, p.331-333.

Wilson, E. O. 1971. The insect societies. Harvard University Press, Cambridge, UK, 548pp. 